\title{
Dark Before Light: Testing the Cosmic Expansion History through the Cosmic Microwave Background
}

\author{
Eric V. Linder ${ }^{1,2}$ and Tristan L. Smith ${ }^{1}$ \\ ${ }^{1}$ Berkeley Center for Cosmological Physics $\&$ Berkeley Lab, \\ University of California, Berkeley, CA 94720, USA \\ ${ }^{2}$ Institute for the Early Universe, Ewha Womans University, Seoul, Korea
}

(Dated: November 12, 2018)

\begin{abstract}
The cosmic expansion history proceeds in broad terms from a radiation dominated epoch to matter domination to an accelerated, dark energy dominated epoch. We investigate whether intermittent periods of acceleration are possible in the early universe - between Big Bang nucleosynthesis (BBN) and recombination and beyond. We establish that the standard picture is remarkably robust: observations of anisotropies in the cosmic microwave background exclude any extra period of accelerated expansion between $1 \leq z \lesssim 10^{5}$ (corresponding to $5 \times 10^{-4} \mathrm{eV} \leq T \lesssim 25 \mathrm{eV}$ ).
\end{abstract}

\section{INTRODUCTION}

Remarkably little is known about the detailed expansion history of the universe. While it proceeds from an epoch of radiation domination, to matter domination, both decelerating, to recent acceleration, even in the best constrained periods - Big Bang nucleosynthesis and recombination - our specific knowledge of the scale factor as a function of time, $a(t)$, is modest. Limits exist on the magnitude of the expansion rate, or Hubble parameter $H=d \ln a / d t$, to $\sim 5 \%$ in the best cases [1, 2], but not on its behavior, for example its slope $d \ln H / d \ln a$. The latter quantity is directly related to the total equation of state $w_{\text {tot }}$ of the energy contents and governs whether the deceleration parameter $q=-1-d \ln H / d \ln a=\left(1+3 w_{\text {tot }}\right) / 2$ is positive (decelerating expansion) or negative (accelerating expansion). The uncertainties on $q$ can easily be of order unity hence allowing either sign - for any short period (such as Big Bang nucleosynthesis or recombination) in the early universe.

This article addresses this lacuna in our understanding through investigation of the effects of a period of early acceleration on the cosmic microwave background (CMB) anisotropies. This is complementary to the recent analysis of Ref. [3] that put severe limits on acceleration at redshifts $z \approx 2-1000$ from effects on matter perturbation growth.

While knowledge of the detailed expansion history is useful in itself, constraints on an early period of acceleration also impact any attempt to ameliorate the coincidence problem of why acceleration is occurring now by adopting models in which acceleration is a persistent or occasional phenomenon (e.g. [4-6]). Sufficiently tight limits could force us to focus on dynamical mechanisms for causing recent acceleration, or to accept the coincidence.

Other rationales for exploring the expansion history for an additional period of acceleration, generally at much earlier epochs than we consider, include: thermal inflation models, which can solve the moduli problem and impact baryogenesis [7], secondary inflation, which can affect the scalar perturbation tilt and matter power spectrum [8, 9], and multiple inflation, which can put features in the curvature perturbation power spectrum [10], and scalar torsion cosmology [11].

In Sec. II we present the basic characteristics of early acceleration and outline the modifications to the background and perturbation equations necessary for determining the CMB anisotropy spectrum. The physical impact of early acceleration on the CMB is analyzed in Sec. III and the numerical results computed. We conclude with a discussion of the constraints on the duration of such an accelerating expansion in Sec. IV]

\section{ADDING EARLY ACCELERATION}

As discussed in Ref. [3], there exist two ways of obtaining an intermittent period of acceleration: driving the dark energy of state highly negative, $w \ll-1$, called superacceleration, or having a relatively high dark energy density with a fairly negative equation of state $w<-1 / 3$, but then $w$ must go positive in order to reduce the dark energy density and permit matter or radiation to dominate again, called superdeceleration. The first approach was shown not to be viable, especially at high redshifts, due to the intrinsic dynamics of the dark energy density evolution for supernegative equations of state, so we only consider superdeceleration models.

\section{A. Acceleration Model}

To model the dark energy, we take a $\Lambda$ CDM universe that undergoes a finite period of deviation from $w=-1$. The e-fold model of Ref. 12] provides a useful parametrization of such a transition, with one step up to positive $w$ and then a later step back down to -1 . This e-fold Ansatz has the physical advantage of defining a characteristic scale for the rapidity of the transition, in terms of number of e-folds of expansion, and the mathematical advantage of giving an analytic expression for the Hubble parameter. 


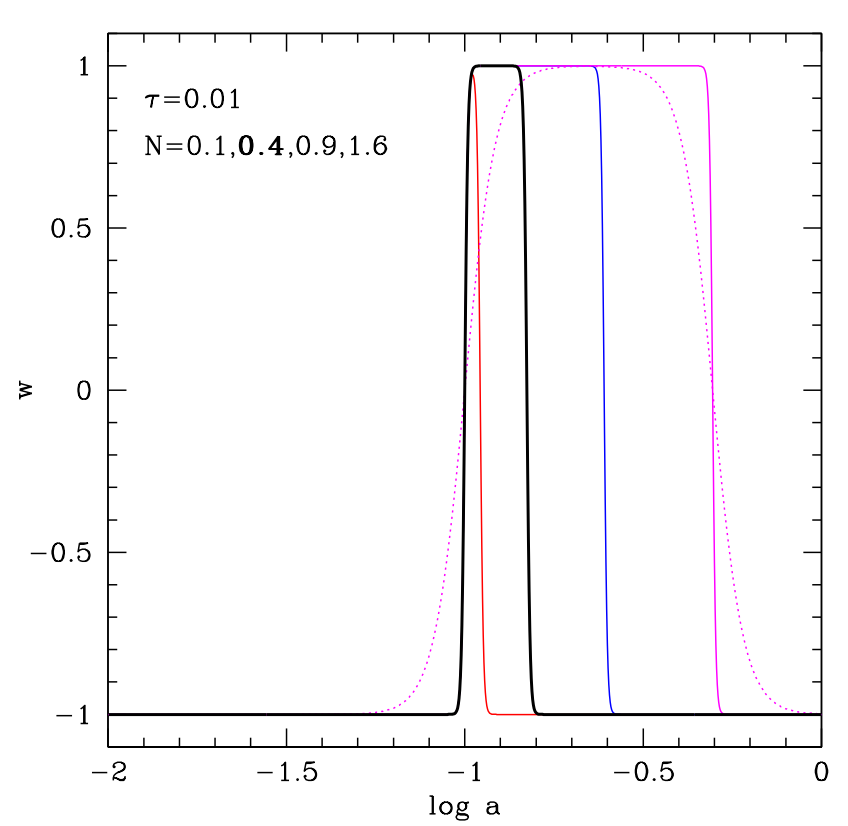

FIG. 1: The double e-fold form for $w(a)$ has useful physical and mathematical properties to describe a step transition. For inverse rapidity $\tau \ll 1$, the transition approximates a step, and for width $N \gtrsim 10 \tau$ the value at the top of the step stays constant for $N$ e-folds. Solid curves all take $\tau=0.01$ and show the behaviors for different values of $N$; the dotted, magenta curve takes $N=1.6$ but for $\tau=0.1$ to show the impact of the rapidity. All curves take the transition up at $a_{t}=0.1$.

The dark energy equation of state as a function of scale factor $a$ is

$$
w(a)=-1+\frac{w_{j}+1}{1+\left(a / a_{d}\right)^{1 / \tau}}-\frac{w_{j}+1}{1+\left(a / a_{t}\right)^{1 / \tau}},
$$

where $w_{j}$ is dark energy equation of state during superdeceleration, $a_{t}$ is the scale factor when $w$ is in the middle of the jump up, $a_{d}=a_{t} e^{N}$ is the scale factor when $w$ is in the middle of the jump back down, $1 / \tau$ is the rapidity of the jumps, i.e. $d w / d \ln a\left(a_{t}\right)=\left(w_{j}+1\right) /(4 \tau)$, and $N$ measures the number of e-folds between the transitions. For small $\tau$, the transition approaches a step function.

At times much earlier than the transition up, $w(a \ll$ $\left.a_{t}\right)=-1$, and at times much later than the transition back down, $w\left(a \gg a_{d}\right)=-1$, while if the transitions are well separated then $w\left(a_{t} \ll a \ll a_{d}\right)=w_{j}$. A true leveling off at $w_{j}$ is achieved for $N \gtrsim 10 \tau$. These behaviors are illustrated in Fig. 1.

The dark energy density evolution follows

$\rho_{w}(a)=\rho_{w, 0}\left(\frac{1+a_{t}^{1 / \tau}}{1+a_{d}^{1 / \tau}}\right)^{3 \tau \Delta w}\left[\frac{1+\left(a / a_{d}\right)^{-1 / \tau}}{1+\left(a / a_{t}\right)^{-1 / \tau}}\right]^{3 \tau \Delta w}$,

where $\Delta w=w_{j}+1$. The dark energy acts like a cosmological constant from the present back to $a \approx a_{d}$, with energy density $\rho_{w, 0}$, then climbs during the step and levels off to a constant $\rho_{w, 0} e^{3 N \Delta w}$ for $a \lesssim a_{t}$. Viewed the other way around, the early dark energy density acts like a larger cosmological constant than usual, allowing early acceleration, then the period of superdeceleration dilutes the density down to the level matching the value today.

It is useful to transform into variables more closely related to the expansion physics such as the scale factor $a_{\text {acc }}$ when the accelerated expansion starts (determined by the condition $w_{\text {tot }}=\sum w_{i}\left(a_{\text {acc }}\right) \Omega_{i}\left(a_{\text {acc }}\right)=-1 / 3$ where the sum runs over all components) and the number of e-folds of accelerated expansion, $N_{\mathrm{acc}}$. Using these as the fundamental parameters, one has

$$
\begin{aligned}
& a_{t}=a_{\mathrm{acc}} e^{N_{\mathrm{acc}}} \\
& a_{d}=a_{\mathrm{acc}} e^{N_{\mathrm{acc}}}\left[\frac{\Omega_{B}\left(1+3 w_{B}\right)}{2 \Omega_{w} a_{\mathrm{acc}}^{3\left(1+w_{B}\right)}}\right]^{1 /\left[3\left(1+w_{j}\right)\right]} \\
& N=\frac{-\left(1+w_{B}\right)}{1+w_{j}} \ln a_{\mathrm{acc}}-\frac{1}{3\left(1+w_{j}\right)} \ln \left[\frac{2 \Omega_{w}}{\Omega_{B}\left(1+3 w_{B}\right)}\right]^{(5)}
\end{aligned}
$$

where $N$ is the number of e-folds of the superdeceleration period, $\Omega_{i}$ is the fractional energy density in component $i$, and a subscript $B$ denotes the dominant component excepting dark energy (e.g. $w_{B}=1 / 3$ when radiation dominates over matter, $w_{B}=0$ when matter dominates over radiation).

The necessary condition to have any acceleration is

$$
N>\frac{1}{3\left(1+w_{j}\right)} \ln \left[\frac{\Omega_{B}\left(1+3 w_{B}\right)}{2 \Omega_{w} a_{t}^{3\left(1+w_{B}\right)}}\right] .
$$

For prerecombination acceleration, $a_{t}$ will be very small and one would need more than 3 e-folds of superdeceleration to allow any acceleration at all. Generally the number of e-folds of superdeceleration is much larger than the number of e-folds of acceleration; it is this that imposes strong constraints on early acceleration from effects on the CMB.

Of course we need not restrict ourselves purely to testing for early acceleration. A modification of the expansion history that is insufficient to give acceleration, i.e. where the early dark energy density is not quite high enough and $N_{\text {acc }}<0$, can still affect CMB observations. We therefore will also be interested in the deviation from the standard expansion history

$$
R=\frac{\Delta H^{2}}{H_{\mathrm{std}}^{2}} \approx \frac{\rho_{w}(a)}{\rho_{B}(a)}=\frac{1+3 w_{B}}{2}\left(\frac{a}{a_{\mathrm{acc}}}\right)^{3\left(1+w_{B}\right)} r(a),
$$

where again $B$ denotes the main background component (radiation or matter), the second equality holds when one component dominates in the standard scenario, and $r\left(a<a_{t}\right)=1, r\left(a_{t}<a<a_{d}\right)=\left(a / a_{t}\right)^{-3 \Delta w}$. We can ask when $R$ exceeds some value, $R_{*}=0.1$ or 1 say. The number of e-folds $N_{*}$ when $R>R_{*}$, and the maximum 
deviation $R_{\max }$, are given by

$$
\begin{aligned}
N_{*} & =\frac{1+w_{j}}{3\left(1+w_{B}\right)\left(w_{B}-w_{j}\right)} \ln \frac{R_{*}}{R_{\max }} \\
R_{\max } & =\frac{1+3 w_{B}}{2} e^{3 N_{\text {acc }}\left(1+w_{B}\right)} .
\end{aligned}
$$

In the analysis we take $w_{j}=+1$, the maximum value consistent with a canonical scalar field, which gives the most conservative case in that this permits the shortest period of superdeceleration for a given acceleration. For the same reason we adopt $\tau \ll 1$. Therefore there are two free parameters: one describing the time when the transition occurs, and one for its duration. These determine the physical effects on the CMB anisotropy power spectrum.

\section{B. Perturbation Equations}

When modifying the expansion history through an additional, dark component one must also account for modifications to the perturbed quantities in order to ensure energy momentum conservation. We include the generalized dark fluid following Ref. [13], taking it to be a minimally coupled, canonical scalar field. Then the density and velocity perturbations to this fluid in synchronous gauge, for each Fourier mode $k$, take the form

$$
\begin{aligned}
\frac{\delta_{g}^{\prime}}{1+w} & =-\frac{\mathcal{H}(1-w)}{k(1+w)} \delta_{g} \\
& -\left[1+9 \frac{\mathcal{H}^{2}}{k^{2}}(1-w)+3 \frac{\mathcal{H} w^{\prime}}{k(1+w)}\right] v_{g}-\frac{1}{2} h^{\prime}, \\
v_{g}^{\prime} & =-\frac{\mathcal{H}}{k} v_{g}+\frac{1}{1+w}\left[\delta_{g}+3 \frac{\mathcal{H}}{k}(1+w) v_{g}\right],
\end{aligned}
$$

where $h$ is one of the metric perturbations in synchronous gauge (see, e.g., [14] ), $\mathcal{H}=a^{-1} d a / d \eta, \delta_{g}$ is the perturbed energy density of the dark fluid, $v_{g}$ is the velocity perturbation, and the prime indicates a derivative with respect to $k \eta$ where $\eta$ is the conformal time.

The metric perturbation $h$ couples these equations to the matter and radiation perturbations. We take adiabatic initial conditions for the dark fluid. Note the presence of the $w^{\prime}$ term; we have checked explicitly that the sharp, but brief, transitions we use do not affect the results. As we vary the inverse rapidity from $\tau=0.05$ to 0.02 or 0.1 , say, the resulting power spectra remained robust. We coded CAMB [15] with the modified background expansion and modified perturbation equations and investigated the effects on the CMB power spectrum.

\section{CMB POWER SPECTRUM AND ACCELERATION}

There are three main cases for the impact of early acceleration. First we will consider the effects of a modified expansion history when it occurs after recombination (similar to Ref. [3] but here with respect to the CMB rather than matter growth). In this case the dominant effect on the CMB power spectrum will be a shift in the angular diameter distance to the surface of last scattering (leading to a shift in the location of the acoustic peaks in the power spectrum) as well as an extra bump at lower multipoles due to a modified integrated Sachs-Wolfe effect. Second we will consider the effect of a modification occurring before recombination. In this case the evolution of the photon perturbations is altered, leading to an enhancement of power on small scales. The third case is when the altered expansion history significantly overlaps the recombination period, and is basically a superposition of the first two cases.

\section{A. General Properties}

Before exploring the individual cases, let us discuss some general influences of modified expansion on the CMB. First, consider the range of redshift that is accessible through observations of the CMB. A measurement of the CMB anisotropy for a given multipole $\ell$ corresponds to a physical wave-number $k$ through the scaling $k \eta_{0} \sim \ell$, where $\eta_{0} \sim 10^{4} \mathrm{Mpc}$ is the conformal time today. A modified expansion history will affect most significantly the evolution of the perturbations for modes that are within the horizon during the modified expansion. Since modes are within the horizon when $k \eta(z) \gtrsim 1$, we expect that only those multipoles with $\ell \gtrsim \eta_{0} / \eta\left(z_{\text {mod }}\right)$ will be significantly modified. Given that observations of the primordial CMB can only be made up to $\ell_{\max } \sim 3000$ before secondary anisotropies become significant, by using the CMB we can expect to be sensitive to modifications in the expansion history up to $z_{\max } \sim 10^{5}$. As we will see, modifications to the expansion history at larger redshifts will have a diminishing effect on the CMB anisotropies.

Figure 2 shows the rough range of multipoles over which modifications will show up in the CMB power spectrum. In fact, effects can appear at somewhat smaller multipoles as well since the enhanced energy density and hence Hubble parameter persists to later times, i.e. smaller redshifts, extending the multipole range for fixed $z$ or extending the redshift sensitivity for fixed $\ell$. From Eqs. 8 and 9 one finds that for $N_{\text {acc }}=0.1$ and $R_{*}=0.5$, say, the effective redshift is lowered by a factor 2 , hence a modification starting at $z=10^{5}$ actually affects down to $\ell \approx 1000$, or conversely sensitivity to $\ell=3000$ actually extends to redshifts $z \approx 2 \times 10^{5}$. For $N_{\text {acc }}=1$, the maximum redshift probed becomes $z \approx 3 \times 10^{6}$.

Another generic effect is on the geometric information in the CMB from the distance scales involved. The location of the acoustic peaks of the $\mathrm{CMB}$ anisotropies is determined by the simple relation $k_{A}=\pi / r_{s}\left(\eta_{\mathrm{rec}}\right)$ [16] where $r_{s}$ is the sound horizon. (We discuss phase factors from potential driving in Sec. IIIC they are unimportant for the following scaling argument.) We can approximately write $r_{s} \sim \eta_{\text {rec }}$, where $\eta_{\text {rec }}$ is the conformal 


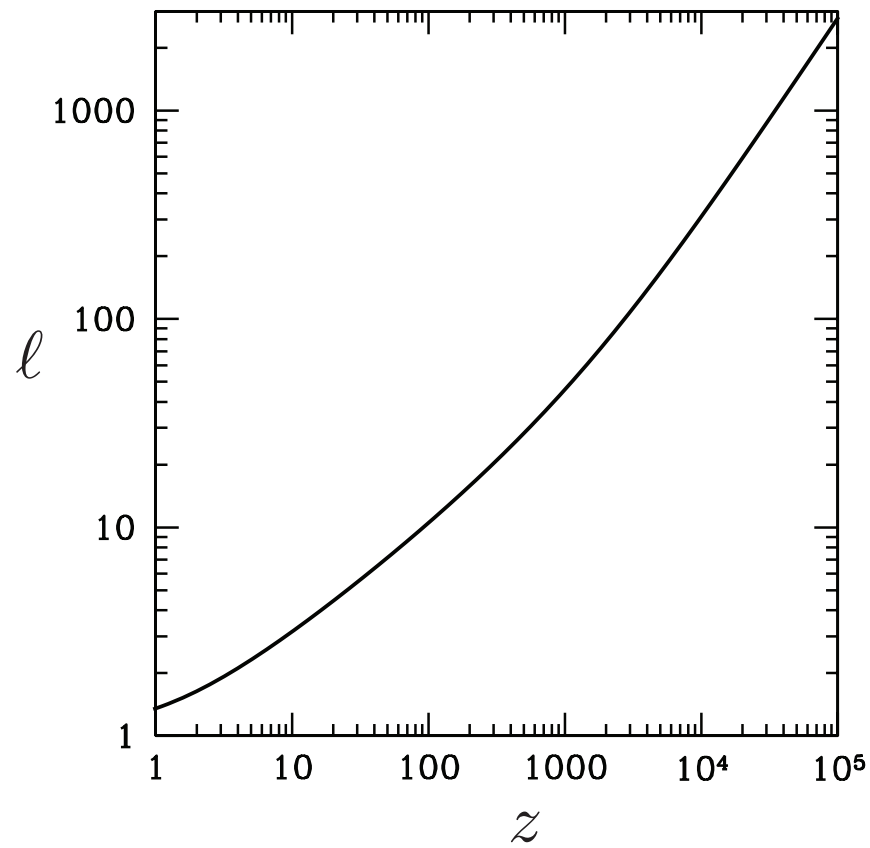

FIG. 2: The minimum multipole affected by modified expansion is plotted as a function of redshift in the standard $\Lambda$ CDM cosmology. Only multipoles above this had associated wavenumbers $k$ within the horizon at redshift $z$. For measurements of the primary CMB anisotropy up to $\ell_{\max } \sim 3000$, say, one can therefore probe the expansion history out to $z_{\max } \sim 10^{5}$.

time at recombination, so that the location of the peaks in harmonic space scales as $\ell_{p} \propto \eta_{0} / \eta_{\text {rec }}$. Since $\eta$ is inversely proportional to $H$ then an increase in $H$ (e.g. due to the extra dark energy density needed for early acceleration) leads to a decrease in $\eta$. If $H$ is increased after recombination then $\eta_{0}$ decreases whereas $\eta_{\text {rec }}$ remains unchanged and the peaks move to smaller $\ell$; if $H$ is increased before recombination then $\eta_{\text {rec }}$ is decreased whereas $\eta_{0}$ remains nearly unchanged (since it receives most of its contribution from late times), leading to a shift in the peaks towards larger values of $\ell$.

Finally, for the models that we consider the redshift of recombination $z_{\text {rec }} \approx 1090$ is left nearly unchanged. We can understand this by noting the redshift of recombination is approximately determined when the expansion rate is equal to the interaction rate between photons and free electrons,

$$
X_{e} n_{b} \sigma_{T}=H
$$

where $n_{b}$ is the baryon number density, $X_{e}$ the ionization fraction, and $\sigma_{T}$ the Thomson scattering cross section. If $H$ is larger at a given redshift $z$ during recombination then due to the detailed balance equations the free electron fraction will similarly be larger (the faster the universe is expanding at a given redshift the harder it will be for free electrons to combine with protons). This balance (described in more detail in [17] for the standard model and in [18] for general $H$ ) leads to $z_{\text {rec }}$ remaining nearly unchanged.

\section{B. Post-Recombination Effects}

Modifying the expansion history in the postrecombination phase produces two distinct physical effects on the CMB temperature power spectrum. Both arise from the change in expansion history rather than the photon perturbation per se. One physical effect is that modifying the expansion history changes the angular diameter distance to the surface of last scattering, leading to a shift in the location of the acoustic peaks in the power spectrum, as already discussed. Note that because the modification is strictly post-recombination, no change occurs in the sound horizon length, so the shift is given purely by the angular distance change.

For a sharp transition lasting a small fraction of an e-fold, one can calculate the effect analytically:

$$
\frac{\delta d_{\mathrm{lss}}}{d_{\mathrm{lss}}} \approx-\frac{3}{7} \frac{\Omega_{m}}{\Omega_{w}} \frac{1}{\sqrt{\Omega_{m}} d_{\Lambda}} a_{d}^{7 / 2} N\left(1+w_{j}\right),
$$

where $d_{\Lambda}$ is the distance to CMB last scattering in the standard, no transition case. The main effect is from the high dark energy density before the transition, rather than the transition (period with $w_{j}=1$ ) itself. Note that for small $N$ the key parameter, other than the timing of the transition $a_{d}$, is an "equivalent width" $N\left(1+w_{j}\right)$ or area of the deviation from $w=-1$ in $\ln a$. For large $N$, the shift increases more rapidly than in Eq. (13).

Observations made by WMAP [19] have constrained the location of the first acoustic peak to within $0.3 \%$. As a first look, nearly analytically, we can use this measurement to exclude regions of the $N_{\text {acc }}-a_{\text {acc }}$ parameter space, fixing all recent universe parameters and testing for post-recombination expansion modifications. As seen in Fig. 3. almost no early acceleration is permitted, except for a tiny region near $z \approx 10^{3}$ amounting to less than 0.14 e-folds of acceleration. The Planck satellite determination of the acoustic peak location to $0.09 \%$ would completely rule out early acceleration (recall that there is a modification of the Hubble parameter due to excess energy density even if it does not rise to the level of causing acceleration). Apart from $N_{\text {acc }}$, one can also directly limit the modification to the expansion as given by the dashed (WMAP level) and dotted (Planck level) curves in Fig. 3. We revisit the constraints rigorously in Sec. IIIE.

The second effect is that the change in the expansion rate leads to the decay of the gravitational potentials, giving rise to a modified integrated Sachs-Wolfe (ISW) effect. The location of the extra ISW power is related to the time of the expansion modification, with earlier transitions leading to an effect at higher multipoles (where cosmic variance is not as severe).

Figure 4 shows the changes induced in the CMB power spectrum from a period of early acceleration lasting for $N_{\text {acc }}=0.1$ e-folds, with the top panels corresponding 


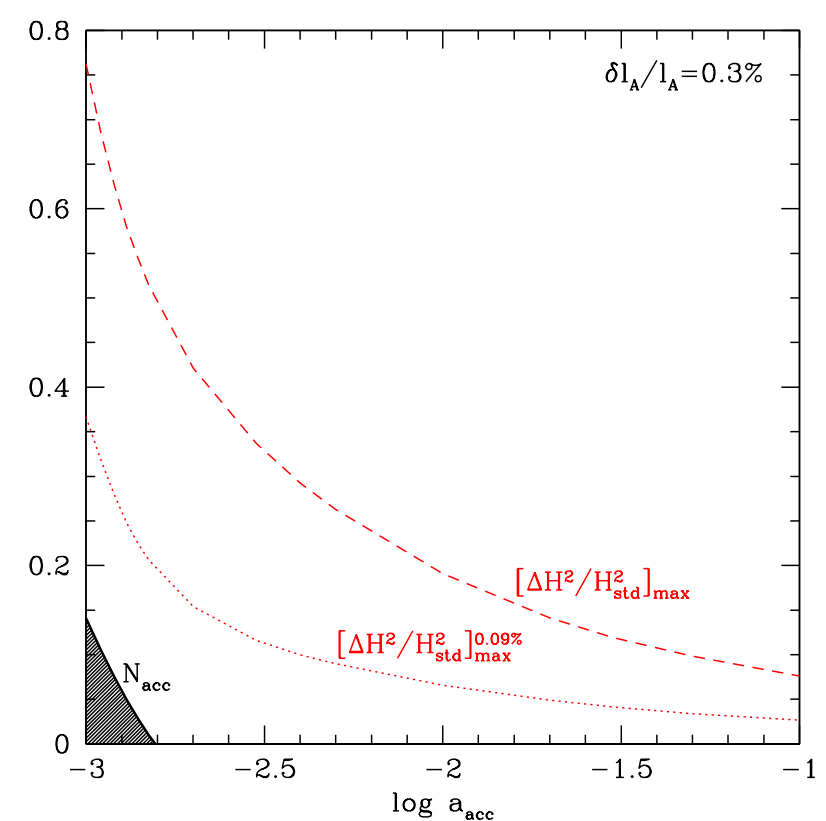

FIG. 3: An upper limit on the number of e-folds of early acceleration in the post-recombination case can be derived by requiring that the acoustic peak location $l_{A}$ be shifted by less than $0.3 \%$ (current WMAP accuracy) by effects on the distance to last scattering $d_{\mathrm{lss}}$. This leaves only the tiny shaded corner of parameter space, with $N_{\text {acc }}<0.14$ at $z \approx$ 1000. Alternately, one could ask how large a deviation is allowed from the standard Hubble parameter (squared). This is restricted at the tens of percent level (red dashed curve), or a factor of 2-3 less for $0.09 \%$ or Planck level constraints on $l_{A}$ (red dotted curve). In fact, constraints are even more severe if one takes into account ISW and other CMB power spectrum information, although they loosen if one marginalizes over recent universe parameters such as $\Omega_{m}$ (see Fig. 5 for limits taking both these effects into account).

to the post-recombination transition discussed in this section. One can clearly see the rise in ISW power at low multipoles (with $\ell$ increasing as $z_{\text {acc }}$ does). The geometric shift in the acoustic peak locations to smaller multipoles is also visible, and this shift gets reversed to higher multipoles for transition pre-recombination. We discuss the other effects from pre-recombination acceleration, and the right panels displaying the potential perturbations, in the next subsection.

\section{Pre-Recombination effects}

Modifying the expansion history pre-recombination leads to a modification of the observed power spectrum on small scales, due mainly to the influence on the photon perturbations between when a given wavemode enters the horizon and recombination. There is also a geometric shift but since the sound horizon length gets its main contribution from near recombination, a sufficiently early transition has little effect on the acoustic scale (the shift goes roughly as $a_{t} N\left(1+w_{j}\right)$ for $\left.N \ll 1\right)$.

Heuristically, one can view the enhancement of power on small scales due to the decay of the potentials in terms of a compressed photon fluid rebounding while feeling a reduced gravitational restoring force [16]. We briefly give a more detailed explanation now.

While the photons are tightly coupled to the baryons they follow a driven harmonic oscillator equation [16]

$$
\left\{\frac{d^{2}}{d x^{2}}+1\right\}\left[\delta_{\gamma}+\Phi\right]=2 \Phi,
$$

where $x \equiv k \eta / \sqrt{3}, \Phi$ is the potential, and we have neglected the time dependence of the sound speed of the photon-baryon fluid and neglected anisotropic stress and dropped the damping terms since they are negligible to a first approximation. The solution which corresponds to adiabatic initial conditions gives

$$
\begin{aligned}
\delta_{\gamma}(x)+\Phi(x)= & {\left[\delta_{\gamma}(0)+\Phi(0)\right] \cos (x) } \\
& +2 \int_{0}^{x} d x^{\prime} \Phi\left(x^{\prime}\right) \sin \left[x-x^{\prime}\right]
\end{aligned}
$$

The modified expansion history, as well as perturbations in the dark fluid that driving the modification, leads to a change in the potential $\Phi$ (as is well known at late times in $\Lambda \mathrm{CDM}$ cosmologies). This in turn drives the photon perturbations to a larger amplitude.

The CMB power spectrum for the pre-recombination modification case is shown in the bottom panels of Fig. 4. For $z_{\text {acc }}=10^{3}$ the modified expansion history leads to an increased early ISW which in turn increases the height of the first peak. The heights of the following peaks have increased due to the decrease in the amplitude of the potential, shown in the right panel. The $z_{\text {acc }}=10^{4}$ case clearly shows that with the modified expansion history the location of the peaks shift toward higher $\ell$. As described before this is due to the decrease in the sound horizon at decoupling because of the increased Hubble parameter. For modifications at such high $z$ there is little effect on the ISW contribution.

Moving to even higher redshift weakens all the effects since the sound horizon scale is mostly governed by conditions near recombination and early modifications affect the potential driving of perturbation modes at high $\ell$ where they are more strongly damped. Observations of the small scale CMB with instruments such as the Atacama Cosmology Telescope (ACT) 21] or the South Pole Telescope (SPT) [22] allow us to constrain the expansion history up to $z \sim 10^{5}$ since they measure the primordial anisotropies to $\ell \sim 3000$ (see Fig. 2). Current constraints allow less than 0.1 e-folds of accelerated expansion at $z_{\text {acc }}=10^{5}$. Planck 23] will be cosmic variance limited out to $\ell \approx 2500$ and delivers tighter constraints as we discuss in Sec. IIE. 

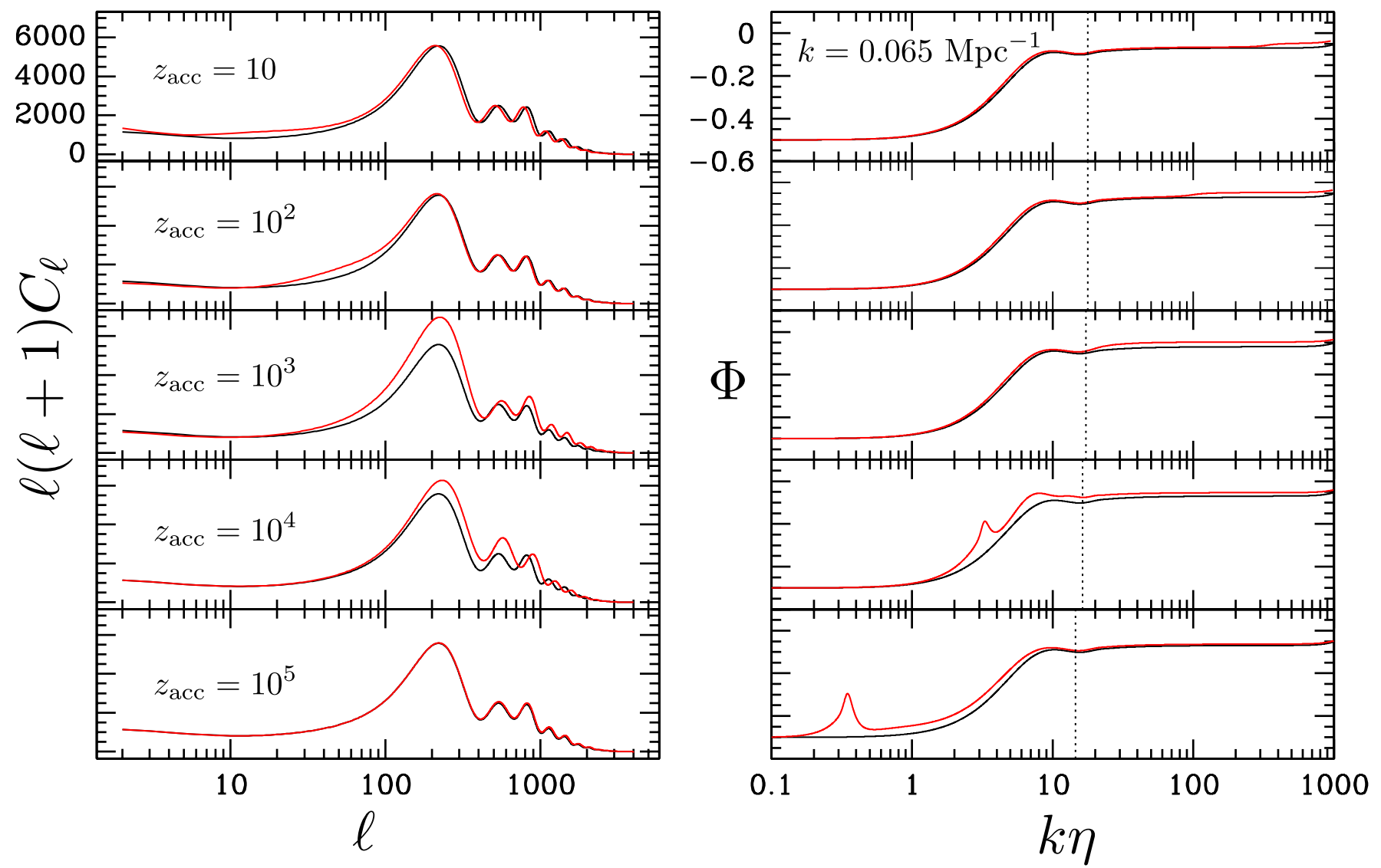

FIG. 4: Left: The CMB temperature power spectrum is plotted for the standard expansion history (black curves) and a modified expansion history (light, red curves). In all panels a period of accelerated expansion starts at redshift $z_{\text {acc }}$ and lasts for 0.1 e-folds. Right: The evolution of the curvature potential corresponding to each case in the left panels is shown for the mode with $k=0.065 \mathrm{Mpc}^{-1}$. The vertical dotted lines show the value of $k \eta_{\mathrm{rec}}$, and one sees how the potential decay occurs post-recombination for the top panels and pre-recombination for the bottom panels. For $z_{\text {acc }}<z_{\text {rec }}$ the power spectrum peaks are shifted slightly towards lower $\ell$ since the angular diameter distance to the surface of last scattering decreases. In addition to this the modified expansion history causes an evolution in the potentials, as seen on the right, leading to an additional integrated Sachs-Wolfe bump in the power spectrum (most visible in the $z_{\text {acc }}=10$ case). As discussed further in the text, when $z_{\text {acc }} \gtrsim z_{\text {dec }}$ two different effects are important. The sound horizon at decoupling is decreased leading to a shift of the peaks to higher $\ell$ (most visible in the $z_{\text {acc }}=10^{4}$ case). In addition to this, the right panels show that the amplitude of the potential is decreased, which leads to a corresponding increase in the amplitude of the photon perturbations seen in the left panels.

\section{Near-recombination effects}

For a transition near recombination, the effects are basically a superposition of the pre-recombination and postrecombination ones, since the influence of the extra dark energy density persists from before to after recombination. One other, more minor effect enters on very small scales. The diffusion of photons leads to a damping of perturbations $\sim e^{-\ell^{2} / \ell_{D}^{2}}$ below a scale $\ell_{D}$. A simple argument (see, e.g., [20]) shows that this scale goes as

$$
\ell_{D} \sim \eta_{0} \sqrt{\frac{X_{e} n_{b} \sigma_{T} a}{\eta_{\mathrm{rec}}}} .
$$

An increased Hubble parameter before recombination will lead to a decrease in $\eta_{\text {rec }}$, an increase in $\ell_{D}$, and hence an increase in the amplitude of perturbations on small scales. This adds somewhat to the enhancement due to the potential decay.

\section{E. Constraints on Early Acceleration}

For any particular redshift in cosmic history, we can ask how much acceleration is permitted, i.e. how many e-folds $N_{\text {acc }}$ can occur starting at that time. Carrying out a Fisher matrix analysis using the CMB temperature power spectrum only, with Planck sensitivity, we find that no extra period of acceleration is permitted for $z_{t}<2 \times 10^{5}$ at more than $99 \%$ confidence level. However the constraints rapidly weaken for higher redshifts, with acceleration being allowed for $z_{t}>3 \times 10^{5}$. The 


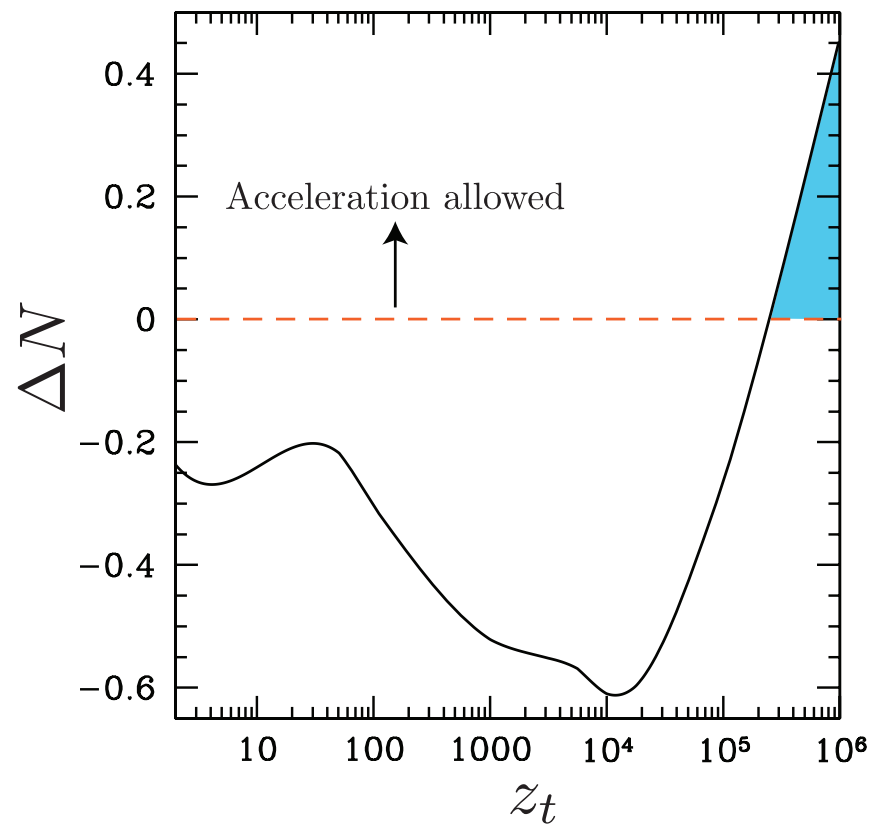

FIG. 5: The curve gives the $95 \% \mathrm{cl}$ limit, assuming Planck CMB temperature sensitivity, on $\Delta N$, the number of superdecelerating e-folds relative to that required for acceleration. Only in the upper right shaded region does the bound allow acceleration, $\Delta N \geq 0$. For redshifts $z_{t}<2.5 \times 10^{5}$ early acceleration is ruled out.

results are displayed in Fig. 5 With WMAP temperature sensitivity one cannot exclude extra acceleration at $z_{t}>4 \times 10^{4}$. Inclusion of polarization data, or matter power spectra, would further tighten the bounds. The main point is that no additional period of acceleration is allowed to ameliorate the coincidence problem of current acceleration.

\section{CONCLUSIONS}

A basic question to ask about the cosmic expansion history is whether it is really as simple as the standard picture of a radiation dominated era giving way to matter domination and later the current epoch of acceleration. Could there have been acceleration earlier, other than high energy inflation, disrupting these eras? We demon- strate that the cosmic microwave background data delivers clear support of the standard picture, forbidding acceleration between $1 \lesssim z \lesssim 2 \times 10^{5}$.

The model chosen for early acceleration is the most conservative case in the sense that we dilute the effects of the extra energy density as quickly as possible $\left(w_{j}=\right.$ 1), at least within the quintessence framework. Even so, no solution to the coincidence problem is possible by taking acceleration to be an occasional phenomenon. Our current epoch of acceleration appears to be essentially unique within the last factor 100,000 of cosmic expansion, or the energy range $5 \times 10^{-4} \mathrm{eV} \lesssim T \lesssim 25 \mathrm{eV}$.

Observations of the CMB are sensitive to acceleration and energy density through a range of physical effects, at various epochs and angular scales. Acceleration gives extra power to the integrated Sachs-Wolfe effect and the small scale photon perturbations through decay of potentials, while extra energy density affects geometric distance factors, shifting the acoustic peaks to larger or smaller scales depending on whether the modification is post- or pre-recombination.

For the epochs between $z \approx 10^{5}-10^{9}$, and between primordial nucleosynthesis and inflation, no observational probes constrain the expansion history. Detection and characterization of dark matter may eventually offer one window on these epochs, as can theoretical models of baryogenesis or the production of axions (see, e.g., 24]). Large swaths of early cosmic history remain dark, and possibly filled with dark energy.

\section{Acknowledgments}

We thank Ed Copeland, Marina Cortês, Sudeep Das, Roland de Putter, Kim Griest, and Wayne Hu for useful discussions. EL thanks the Centro de Ciencias Pedro Pascual in Benasque, Spain and TLS thanks the Institute for the Early Universe, Ewha University, Korea for hospitality. This work has been supported in part by the Director, Office of Science, Office of High Energy Physics, of the U.S. Department of Energy under Contract No. DE-AC02-05CH11231, and the World Class University grant R32-2009-000-10130-0 through the National Research Foundation, Ministry of Education, Science and Technology of Korea.
[1] S.M. Carroll \& M. Kaplinghat, Phys. Rev. D 65, 063507 (2002) arXiv:astro-ph/0108002

[2] S. Galli, A. Melchiorri, G.F. Smoot, O. Zahn, Phys. Rev. D 80, 023508 (2009) arXiv:0905.1808

[3] E.V. Linder, Phys. Rev. D 82, 063514 (2010) arXiv:1006.4632

[4] S. Dodelson, M. Kaplinghat, E. Stewart, Phys. Rev. Lett. 85, 5276 (2000) arXiv:astro-ph/0002360
[5] K. Griest, Phys. Rev. D 66, 123501 (2002) arXiv:astro-ph/0202052

[6] G. Barenboim, O. Mena, C. Quigg, JCAP 0604, 008 (2006) arXiv:astro-ph/0510178

[7] D.H. Lyth \& E.D. Stewart, Phys. Rev. D 53, 1784 (1996) arXiv:hep-ph/9510204

[8] L. Ackerman, W. Fischler, S. Kundu, N. Sivanandam, arXiv:1007.3511 
[9] S. Ilic, M. Kunz, A.R. Liddle, J.A. Frieman, Phys. Rev. D 81, 103502 (2010) arXiv:1002.4196

[10] P. Hunt \& S. Sarkar, Phys. Rev. D 76, 123504 (2007) arXiv:0706.2443.

[11] K-F. Shie, J.M. Nester, H-J. Yo, Phys. Rev. D 78, 023522 (2008) arXiv:0805.3834

[12] E.V. Linder \& D. Huterer, Phys. Rev. D 72, 043509 (2005) arXiv:astro-ph/0505330

[13] W. Hu, Astrophys. J. 506, $485 \quad$ (1998) arXiv:astro-ph/9801234.

[14] C. P. Ma and E. Bertschinger, Astrophys. J. 455, 7 (1995) arXiv:astro-ph/9506072

[15] http://camb.info A. Lewis \& A. Challinor, Astrophys. J. 538, 473 (2000) arXiv:astro-ph/9911177.
[16] W. Hu and N. Sugiyama, Astrophys. J. 444, 489 (1995) arXiv:astro-ph/9407093

[17] B.J.T. Wyse \& R.F.G. Jones, Astr. Astrophys. 149, 144 (1985)

[18] E.V. Linder, arXiv:astro-ph/9712159

[19] N. Jarosik et al., arXiv:1001.4744

[20] S. Dodelson, Modern Cosmology, (Academic Press, 2003)

[21] S. Das et al., arXiv:1008.0847

[22] M. Lueker et al., Astrophys. J. 719, 1045 (2010) arXiv:0912.4317

[23] http://planck.esa.int

[24] D. Grin, T. L. Smith, M. Kamionkowski, Phys. Rev. D 77, 085020 (2008) arXiv:0711.1352 